\title{
The Synchronous Presence of Multiple Myelomas and Other Primary Malignant Tumors: Case Series with Literature Review
}

This article was published in the following Dove Press journal: Cancer Management and Research

\author{
Ning $L i^{1, *}$ \\ Xiyang Liu ${ }^{2} *$ \\ Yongping Song ${ }^{2}$ \\ Suxia Luo' \\ Baijun Fang ${ }^{2}$ \\ 'Department of Oncology, Henan \\ Cancer Hospital, Henan Cancer Hospital \\ Affiliated to Zhengzhou University, \\ Zhengzhou University, Zhengzhou, \\ People's Republic of China; ${ }^{2}$ Henan \\ Institute of Haematology, Department of \\ Hematology, Henan Cancer Hospital, \\ Henan Cancer Hospital Affiliated to \\ Zhengzhou University, Zhengzhou \\ University, Zhengzhou, People's Republic \\ of China
}

*These authors contributed equally to this work
Correspondence: Baijun Fang Henan Institute of Haematology,

Department of Hematology, Henan

Cancer Hospital, Henan Cancer Hospital

Affiliated to Zhengzhou University,

Zhengzhou University, 127 Dongming

Road, Zhengzhou 450008, People's

Republic of China

Tel +86-37I-655873I8

Fax +86-37I-6596I505

Email bjfdr_sea@I63.com
Objective: The synchronous presence of multiple myeloma (MM) and other primary malignant tumors (PMTs) were rarely reported. This study aimed to analyze several cases of MM and other PMTs in order to improve clinicians' understanding of multiple myeloma (MM) with sMPMTs.

Methods: This study was a retrospective trial. We retrospectively analyzed six cases of the synchronous presence of MM and other PMTs and reviewed the literature to summarize the clinical features and treatment.

Results: The results showed that five cases of immunoglobulin G (IgG) and one case of kappa light chain; D-S stage: six case of stage III; ISS stage: one case of stage I, two cases of stage II, and three cases of stage III; one case each of gastric cancer (pT2N0MO, stage I), breast cancer (pT1bN0M0, stage I), lung cancer (pT1N0M0, stage I), cervical cancer (stage IB2), thyroid cancer (pT1N0M0, stage I), and diffuse large B-cell lymphoma (Ann-Arbor stage II); three of five patients underwent surgery alone, one patient underwent surgery first and then received chemotherapy at the time of pleural metastasis and the other patient only received radiotherapy; two patients were still alive, three died of progression of $\mathrm{MM}$, and one died of lung cancer. The median survival time was 33.5 months (95\% CI, 14.17 to 59.5 months).

Conclusion: The relationship between synchronous MM and other PMTs remains unknown. Clinicians should improve their understanding of MM with sMPMTs by carrying out multidisciplinary collaboration and a patient-oriented approach to optimize treatment and prolong the survival rates of patients.

Keywords: multiple myeloma, primary malignant tumors, synchronous multiple primary malignant tumors, clinical features, treatment

\section{Introduction}

Primary malignant tumor is a malignant tumor caused by changes in the organ itself. Multiple primary malignant tumors (MPMTs) refers to two or more primary malignant tumors in the same individual. ${ }^{1}$ MPMTs may be synchronous or metachronous. The majority of MPMTs that occur in multiple organs are metachronous, while the presence of synchronous MPMTs (sMPMTs) is less common. Although it has been reported that special attention should be given to MPMTs, especially for head, neck, and urinary tumors, ${ }^{1}$ their prevalence is generally very low. Only sporadic cases have been reported concerning multiple myeloma (MM) with sMPMTs. ${ }^{2,3}$ In addition, multiple cycles of combination chemotherapy are necessary for the treatment of newly diagnosed MM. ${ }^{4-8}$ Therefore, deciding when and 
how to start treatment for sMPTS may be difficult. Physicians must balance the risk of re-exacerbating the MM due to insufficient treatment with the risk of exacerbating the untreated sMPMTs. No optimized treatment strategy exists for synchronous MM and other primary malignant tumors (PMTs) so far. This distinction implies an important therapeutic challenge. So therapeutically, a multidisciplinary and patient-oriented approach should be considered.

The synchronous presence of multiple myeloma (MM) and other primary malignant tumors (PMTs) are rarely observed in clinical practice. Furthermore, no optimized treatment strategy exists for synchronous multiple primary malignant tumors (sMPMTs) so for. Therefore, we retrospectively analyzed the data of six cases in patients with synchronous $\mathrm{MM}$ and other primary malignant tumors that were admitted in recent years and reviewed the literature to summarize the clinical features, treatment, and outcomes to increase clinicians' awareness of sMPMTs.

\section{Materials and Methods}

\section{Patients}

From January 2012 to December 2017, six patients who were diagnosed with synchronous $\mathrm{MM}$ and solid tumor were included in this study by searching the medical database records of Hematology Department at the Henan Cancer Hospital affiliated to Zhengzhou University in Zhengzhou (China). The ethics committee of our hospital approved this study and all patients signed informed consent.

\section{Statistical Analysis}

We used the software program SPSS 20.0 (IBM, Chicago, USA) to conduct the statistical analysis. Continuous variables were expressed as mean $\pm \mathrm{SD}$. Discontinuous variables were expressed as a percentage (\%). A value of $\mathrm{P}<0.05$ was considered statistically significant.

\section{Results}

\section{The General Characteristics}

From January 2012 to December 2017, a total of 2000 cases of newly diagnosed cancer in our hospital. The six patients diagnosed with sMPMTs accounted for $0.3 \%$ of newly diagnosed cancer patients during this period in our hospital. The median age of the six patients was 60 years and the median survival time was 33.5 months (95\% confidence interval, 14.17 to 59.5 months). Detailed clinical data are listed in Table 1.

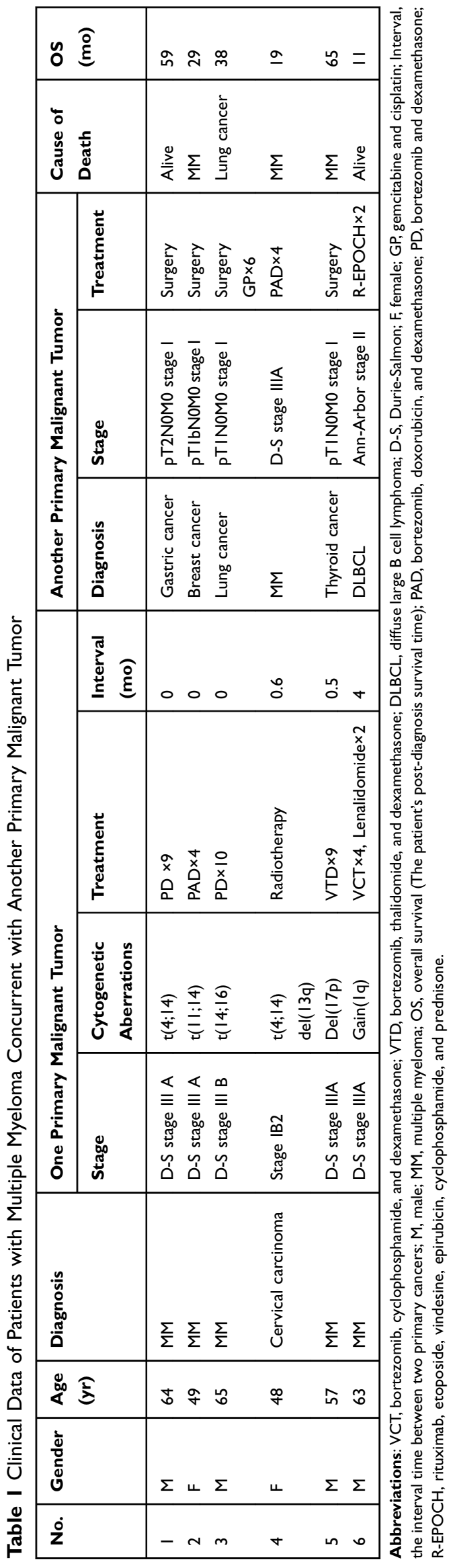




\section{Case I}

A 64-year-old male presented with upper abdominal discomfort and dull pain. Gastroscopy showed gastric cardia polyps (nature to be determined) and a gastric ulcer, which was potentially malignant. A biopsy pathology showed chronic inflammation, acute inflammation, and high atypical hyperplasia in some glands of the gastric cardia and pyloric mucosa, and suspicious focal canceration. At the same time, a pelvic computed tomography (CT) scan showed multiple irregular osteolytic bone destruction of the bilateral iliac and sacral lumbar and myeloma needed to be excluded. Routine blood tests revealed a hemoglobin (HGB) level of $74 \mathrm{~g} / \mathrm{L}$, total leukocyte count of $6.2 \times 10^{9} / \mathrm{L}$, and platelet count of $85 \times 10^{9} / \mathrm{L}$. Blood biochemistry revealed an erythrocyte sedimentation rate (ESR) of $79 \mathrm{~mm} / \mathrm{hr}$ (normal value, $<22 \mathrm{~mm} / \mathrm{hr}$ ), serum globulin of $19.1 \mathrm{~g} / \mathrm{L}$, serum albumin of $37.1 \mathrm{~g} / \mathrm{L}$, serum creatinine of $91 \mu \mathrm{mol} / \mathrm{L}$, serum calcium of $2.59 \mathrm{mmol} / \mathrm{L}$, serum lactate dehydrogenase $(\mathrm{LDH})$ of $171 \mathrm{U} / \mathrm{L}$, and serum beta-2 microglobulin of ( $\beta 2-\mathrm{MG})$ $2.79 \mathrm{mg} / \mathrm{L}$. Serum electrophoresis (EP) and immunoelectrophoresis (IEP) showed an abnormally bowed arc against the anti-kappa light chain and there were no $\mathrm{M}$ bands. A urine immunofixation test was positive for the kappa light chain and free kappa light chain. Bone marrow (BM) aspirate showed an increased population of dysplastic plasma cells (61\%), which were proved to be monoclonal plasma cells by flow cytometry. The sum of these results confirmed two diagnoses: $\mathrm{MM}$ of the kappa light chain-type (DurieSalmon [D-S] stage III A and international staging system [ISS] stage I) and gastric cancer. The patient underwent radical gastrectomy and a jejunal feeding tube placement under general anesthesia after half a month. The postoperative staging was moderately differentiated adenocarcinoma of the cardia (pT2N0M0, stage I) (Figure 1). The PD scheme (bortezomib $1.3 \mathrm{mg} / \mathrm{m}^{2}$ plus dexamethasone $20 \mathrm{mg}$ on days $1,4,8$, and 11) was administered. After two cycles of PD, the patient achieved a very good partial remission (VGPR). After another two cycles, complete remission (CR) was achieved. The PD program was continued for another five cycles as consolidation therapy and multiple reexaminations showed that the patient remained stable. The patient is still alive now and the total survival time has exceeded 59 months.

\section{Case 2}

A 49-year-old female was found to have a right breast mass during a routine health examination. Breast biopsy suggested right breast invasive carcinoma, as seen in Figure 2. Immunohistochemistry showed C-erB-2 (-), CK5/6 (+), EGFR $(++)$, ER (-), Ki-67 (+30\%), nm23 (-), P53 (85\%), P63 (-), and PR (-). The molecular subtype was Basal-like. At the same time, the serum EP and IEP showed abnormally bowed arcs against IgG and anti-kappa light chain. Routine blood tests revealed an HGB level of $83 \mathrm{~g} / \mathrm{L}$, total leukocyte count of $3.1 \times 10^{9} / \mathrm{L}$, and platelet count of $34 \times 10^{9} / \mathrm{L}$. Blood biochemistry revealed an ESR of $73 \mathrm{~mm} / \mathrm{hr}$ (normal value, $<22 \mathrm{~mm} / \mathrm{hr}$ ), serum globulin of $90.9 \mathrm{~g} / \mathrm{L}$, serum albumin of $35.8 \mathrm{~g} / \mathrm{L}$, serum creatinine of $41 \mathrm{umol} / \mathrm{L}$, serum calcium of $1.96 \mathrm{mmol} / \mathrm{L}$, serum LDH of $274 \mathrm{U} / \mathrm{L}$, and serum $\beta 2-\mathrm{MG}$ of $4.25 \mathrm{mg} / \mathrm{L}$. The serum $\mathrm{IgG}$ and serum kappa light chain was $80.52 \mathrm{~g} / \mathrm{L}$ and $27.26 \mathrm{~g} / \mathrm{L}$, respectively. Bone marrow (BM) aspirate demonstrated up to $25 \%$ dysplastic plasma cells, which were proved to be monoclonal plasma cells by flow cytometry. The diagnoses were MM with type of
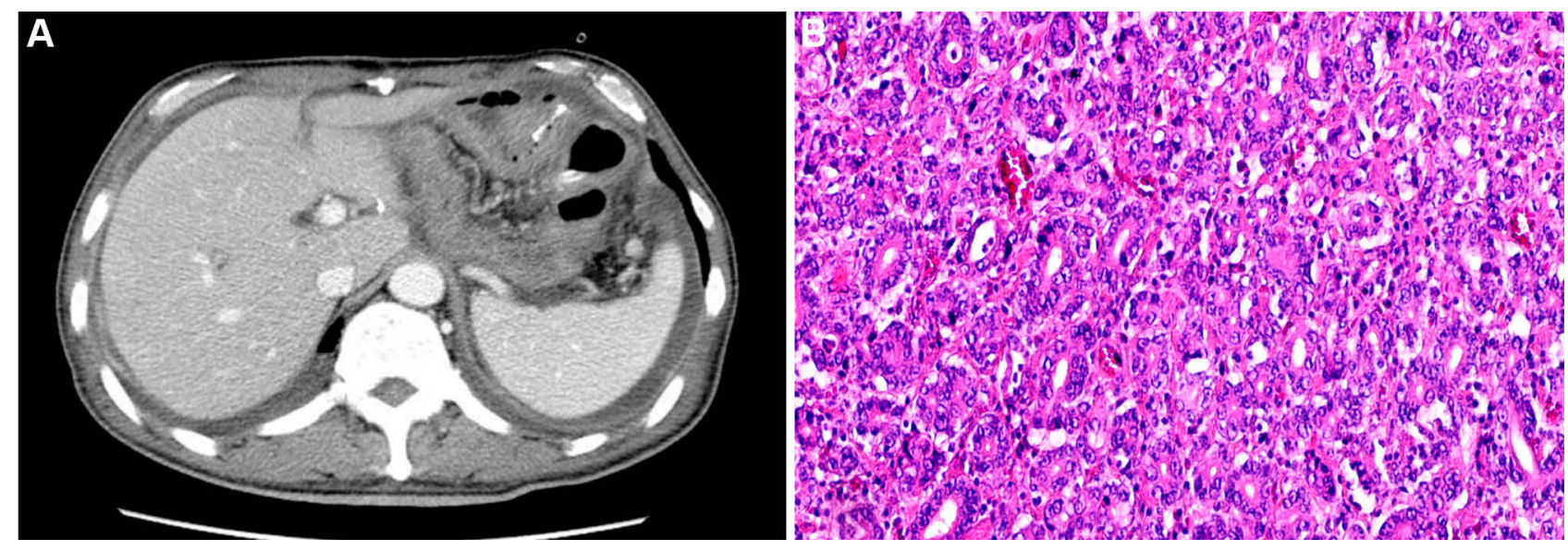

Figure I Case I pathological figures. (A) Computed tomography of gastric stump after gastric cancer surgery. (B) Postoperative pathological biopsy result revealed moderately differentiated adenocarcinoma of cardia (Hematoxylin \& Eosin staining, $\times 20$ ). 

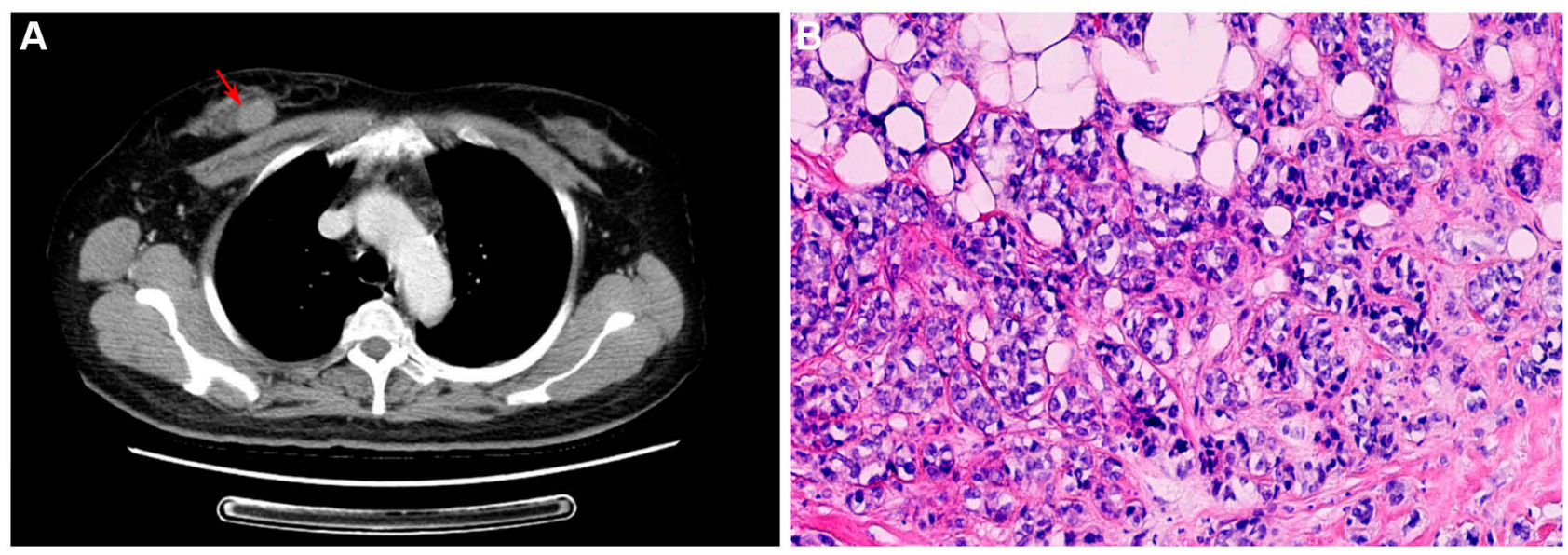

Figure 2 Case 2 pathological figures. (A) Preoperative computed tomography showed a oval nodule in the right breast (arrow). (B) Biopsy revealed breast invasive carcinoma (Hematoxylin \& Eosin staining, $\times 20$ ).

IgG-kappa (D-S stage III A and ISS stage II) and right breast invasive carcinoma. The patient's breast cancer was treated with radical mastectomy and postoperative staging was right invasive ductal carcinoma (pT1bN0M0, stage I). Then the scheme of PAD (bortezomib $1.3 \mathrm{mg} / \mathrm{m}^{2}$ on days $1,4,8$ and 11 , doxorubicin $9 \mathrm{mg} / \mathrm{m}^{2}$ on days $1-4$, and dexamethasone $20 \mathrm{mg}$ on days $1,2,4,5,8,9,11$ and 12 , repeated every 28 days) was applied and the evaluation of the curative effect was partial remission (PR) after four cycles of PAD. Because of intractable severe anemia and thrombocytopenia, the patient refused the follow-up chemotherapy and began to receive $150 \mathrm{mg}$ of thalidomide daily, outside the hospital. Telephone follow-ups revealed that the patient died of MM progression with pulmonary infection and respiratory failure in a local hospital. The patient's post-diagnosis survival time was 29 months.

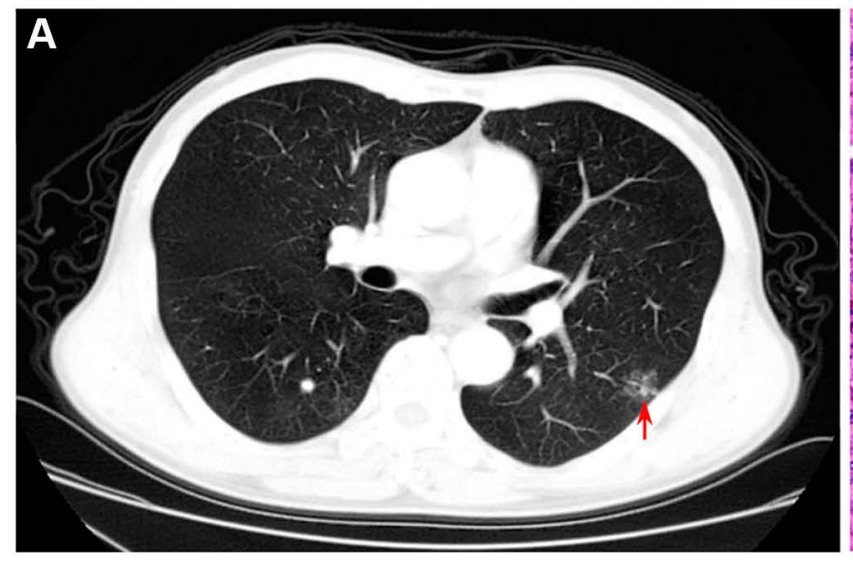

\section{Case 3}

A 65-year-old male presented with pain in the left leg and waist. CT imaging showed a circular and milled vitreous nodule in the left lower lung, which was recommended for puncture and the destruction of the lumbar vertebra and rib bone, which suggested metastasis or MM. Magnetic resonance imaging (MRI) showed multiple areas of destruction in the vertebral body and appendages of T12-L5, S1, S2, and S3. Puncture pathology of the left lung tissue and lumbar paravertebral soft tissue showed moderately differentiated adenocarcinoma (EGFR no mutation, ALK-negative) (Figure 3) and plasmacytoma, respectively. Bone marrow (BM) aspirate demonstrated up to $17 \%$ dysplastic plasma cells, which were proved to be monoclonal plasma cells by flow cytometry. Routine blood tests revealed an HGB level of $93 \mathrm{~g} / \mathrm{L}$, total leukocyte count of $5.7 \times 10^{9} / \mathrm{L}$, and platelet

Figure 3 Case 3 pathological figures. (A) Preoperative computed tomography showed round ground-glass nodule in the left lower lung (arrow). (B) Pathological biopsy revealed left lung moderately-differentiated adenocarcinoma (Hematoxylin \& Eosin staining, $\times 20$ ).

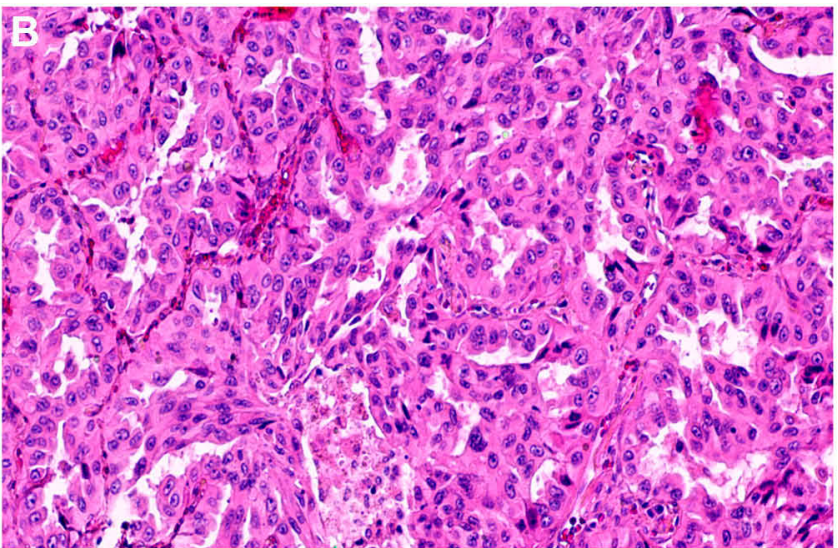


count of $98 \times 10^{9} / \mathrm{L}$. Blood biochemistry revealed an ESR of $97 \mathrm{~mm} / \mathrm{hr}$ (normal value, $<22 \mathrm{~mm} / \mathrm{hr}$ ), serum globulin of $33.2 \mathrm{~g} / \mathrm{L}$, serum albumin of $33.1 \mathrm{~g} / \mathrm{L}$, serum creatinine of $217 \mu \mathrm{mol} / \mathrm{L}$, serum calcium of $2.21 \mathrm{mmol} / \mathrm{L}$, serum LDH of $109 \mathrm{U} / \mathrm{L}$, and serum $\beta 2-\mathrm{MG}$ of $8.55 \mathrm{mg} / \mathrm{L}$. The serum $\mathrm{IgG}$ and serum kappa light chain was $19.76 \mathrm{~g} / \mathrm{L}$ and $8.65 \mathrm{~g} / \mathrm{L}$, respectively. The serum EP and IEP also showed abnormally bowed arcs against IgG and anti-kappa light chain. Positron emission tomography-CT imaging showed irregular nodules in the lower lobe of the left lung consistent with peripheral lung cancer and multiple irregular osteolytic bone destruction in the whole body consistent with changes in MM. The diagnoses were MM with type of IgG-kappa (D-S stage III $\mathrm{B}$ and ISS stage III) and moderately differentiated adenocarcinoma in the left lung. The patient first underwent wedge lobectomy. The postoperative staging was moderately differentiated adenocarcinoma (pT1N0M0, stage I).

Then, the PD scheme (bortezomib $1.3 \mathrm{mg} / \mathrm{m}^{2}$ plus dexamethasone $20 \mathrm{mg}$ on days 1, 4, 8, and 11) was applied and the evaluation of the curative effect was PR after four cycles of PD. Postoperative review in July 2014 suggested pleural metastasis. So the PD and GP schemes (gemcitabine $1000 \mathrm{mg} / \mathrm{m}^{2}$ on days 1 and 8 plus cisplatin $20 \mathrm{mg} / \mathrm{m}^{2}$ on days 1-3) were administered alternately. After alternating the four cycles of PD and GP schemes, the efficacy of MM and lung cancer was evaluated as very good PR and PR, respectively. The patient then alternately received the other two cycles of PD and GP regimens. On the fourth-month posttreatment follow-up, the disease began to progress with metastasis to the liver and the biopsy revealed recurrent adenocarcinoma of the lung. The patient's condition became progressively worsened; he gave up treatment and began experimenting with traditional Chinese medicine and eventually died of lung cancer progression with respiratory failure. The patient's post-diagnosis survival time was 38 months.

\section{Case 4}

A 48-year-old female presented with irregular vaginal bleeding and discomfort of the waist. A gynecologic examination found a cervical swelling and ultrasound revealed a hypoechoic cervix. Cervical biopsy pathology showed poorly differentiated squamous cell carcinoma of the cervix (Figure 4). A concurrent, routine blood test revealed a total leukocyte count of $9.69 \times 10^{9} / \mathrm{L}, \mathrm{HGB}$ of $42 \mathrm{~g} / \mathrm{L}$ and platelet count of $98 \times 10^{9} / \mathrm{L}$. Blood biochemistry revealed a serum albumin of $22.6 \mathrm{~g} / \mathrm{L}$, serum globulin of $101.1 \mathrm{~g} / \mathrm{L}$, serum creatinine of $71 \mu \mathrm{mol} / \mathrm{L}$, serum calcium of $2.11 \mathrm{mmol} / \mathrm{L}$, serum LDH of $155 \mathrm{U} / \mathrm{L}$, and serum $\beta 2-\mathrm{MG}$ of $6.23 \mathrm{mg} / \mathrm{L}$. The serum EP and IEP showed abnormally bowed arcs against IgG and anti-lambda light chain. A chest CT scan showed multiple bone destruction on the right third rib and the eighth and ninth thoracic vertebrae. Bone marrow (BM) aspirate demonstrated up to $36 \%$ dysplastic plasma cells, which were proved to be monoclonal plasma cells by flow cytometry. The diagnoses were cervical squamous cell carcinoma (stage $\mathrm{IB}_{2}$ ) and MM with type of IgG-lambda-type (D-S stage IIIA and ISS stage III). After accepting symptomatic treatments such as blood transfusions and cervical local radical radiotherapy, the PAD regimen (bortezomib $1.3 \mathrm{mg} / \mathrm{m}^{2}$ on days $1,4,8$ and 11 , doxorubicin $9 \mathrm{mg} / \mathrm{m}^{2}$ on days $1-4$, and dexamethasone $20 \mathrm{~m}$ on days $1,2,4,5,8,9$, 11 and 12, repeated every 28 days) was administered. Four cycles later, the efficacy of MM was evaluated as a partial remission. Considering the intractable severe anemia and
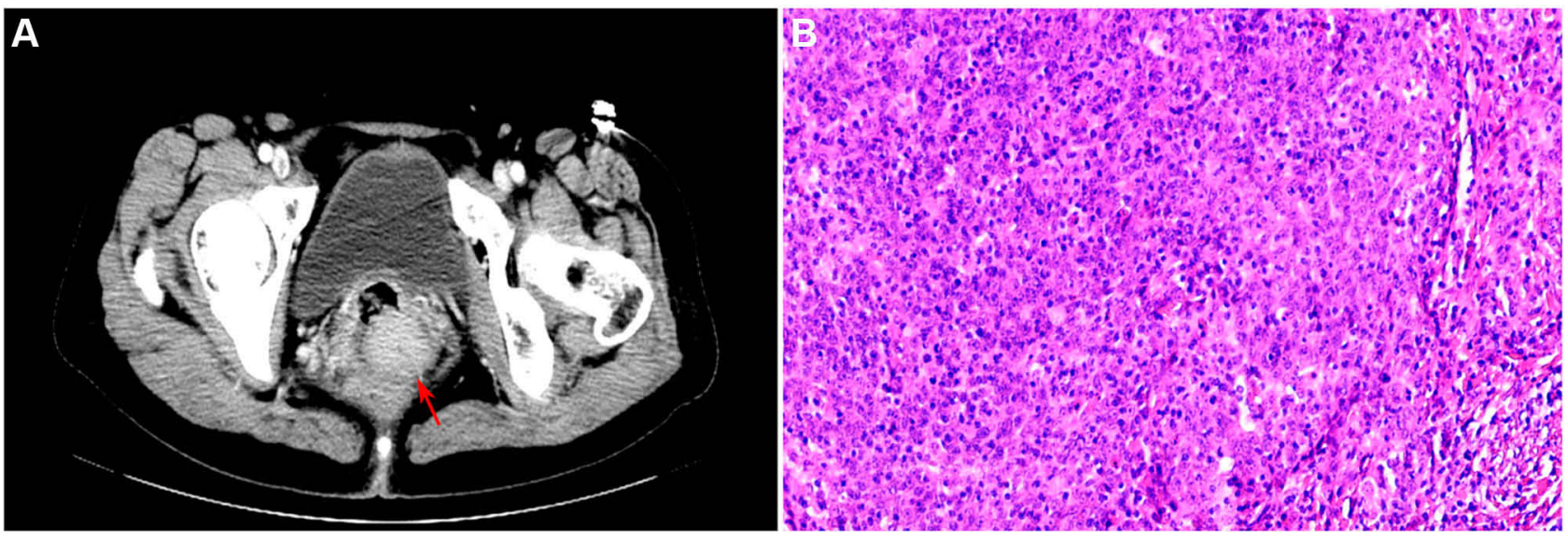

Figure 4 Case 4 pathological figures. (A) Preoperative computed tomography of cervical occupying lesion (arrow). (B) Pathological biopsy result revealed poorlydifferentiated squamous cell carcinoma of the cervix (Hematoxylin \& Eosin staining, $\times 20$ ). 
low platelet level after the above chemotherapy, the patient did not receive follow-up chemotherapy. This patient's progression-free survival duration was three months and she died of MM progression and pulmonary infection at 19 months from diagnosis.

\section{Case 5}

A 57-year-old male presented with pain in the right chest wall and a chest CT scan showed bone destruction on the right fourth rib and the discontinuous cortical bone. Routine blood tests revealed an HGB level of $79 \mathrm{~g} / \mathrm{L}$, total leukocyte count of $4.1 \times 10^{9} / \mathrm{L}$ and platelet count of $117 \times 10^{9} / \mathrm{L}$. Blood biochemistry revealed a serum albumin of $30.8 \mathrm{~g} / \mathrm{L}$, serum globulin of $22.3 \mathrm{~g} / \mathrm{L}$, serum creatinine of $131 \mu \mathrm{mol} / \mathrm{L}$, serum calcium of $2.05 \mathrm{mmol} / \mathrm{L}$, serum LDH of $179 \mathrm{U} / \mathrm{L}$, and serum $\beta 2-\mathrm{MG}$ of $10.95 \mathrm{mg} / \mathrm{L}$. The serum $\mathrm{IgG}$ and serum lambda light chain were $18.08 \mathrm{~g} / \mathrm{L}$ and $4.34 \mathrm{~g} / \mathrm{L}$, respectively. The serum EP and IEP also showed abnormally bowed arcs against IgG and anti-lambda light chain. Bone marrow (BM) aspirate demonstrated up to $42.6 \%$ dysplastic plasma cells, which were proved to be monoclonal plasma cells by flow cytometry. The color Doppler showed a low echo mass in the middle of the left lobe of the thyroid gland of approximately $6 \times 6 \mathrm{~mm}$ with an irregular shape, an unequal edge, and an uneven echo. Punctate foci with strong echo were seen in the mass and no obvious blood flow signals were found in the group of CDFI. Malignant thyroid disease needed to be excluded. Thyroid biopsy revealed epithelial cells with enlarged nuclei and pale staining. False inclusion bodies could be seen in the nucleus and papillary carcinoma was considered (Figure 5). Gene detection of $B$-raf revealed a TA point mutation at the 1799th nucleotide and amino acid variant V600E. Finally, the diagnoses were MM with type of IgG-lambda (D-S stage IIIA and ISS stage III) and papillary thyroid microcarcinoma. One cycle of the VTD scheme (bortezomib $1.3 \mathrm{mg} / \mathrm{m}^{2}$ on days $1,4,8$ and 11 , dexamethasone $20 \mathrm{mg}$ on days $1,2,4,5,8,9,11$ and 12 , and thalidomide $100 \mathrm{mg}$ on days $1-28$, repeated every 28 days) was administered primarily. In the interval of chemotherapy, the patient underwent a roid carcinoma radical operation. The postoperative staging was papillary carcinoma (pT1N0M0, stage I). Then, the patients continued to undergo six cycles of the VTD scheme. After nine cycles of chemotherapy, the curative effect was evaluated as CR. The patient then began to receive $100 \mathrm{mg}$ of thalidomide daily as maintenance treatment outside the hospital. Information received by telephone follow-ups showed that the patient died of severe pulmonary infection with a total survival time of 65 months. His survival duration from the time of diagnosis was 65 months.

\section{Case 6}

A 63-year-old male patient presented with pain in the right hip joint, which intensified after activity. MRI showed that signals from the right femoral neck, middle of the greater and lesser trochanters, and the mid femur were less homogeneous with the formation of a soft tissue mass and edema of the soft tissue. Emission CT showed that bone metabolism in the right femur and the left posterior ninth and tenth ribs was active. Routine blood tests revealed an HGB level of $135 \mathrm{~g} / \mathrm{L}$, total leukocyte count of $6.8 \times 10^{9} / \mathrm{L}$, and platelet count of $106 \times 10^{9} / \mathrm{L}$. Blood biochemistry revealed an ESR of $37 \mathrm{~mm} / \mathrm{hr}$ (normal value, $<22 \mathrm{~mm} / \mathrm{hr}$ ), serum globulin of $28.3 \mathrm{~g} / \mathrm{L}$, serum albumin of $33.1 \mathrm{~g} / \mathrm{L}$, serum creatinine of 74
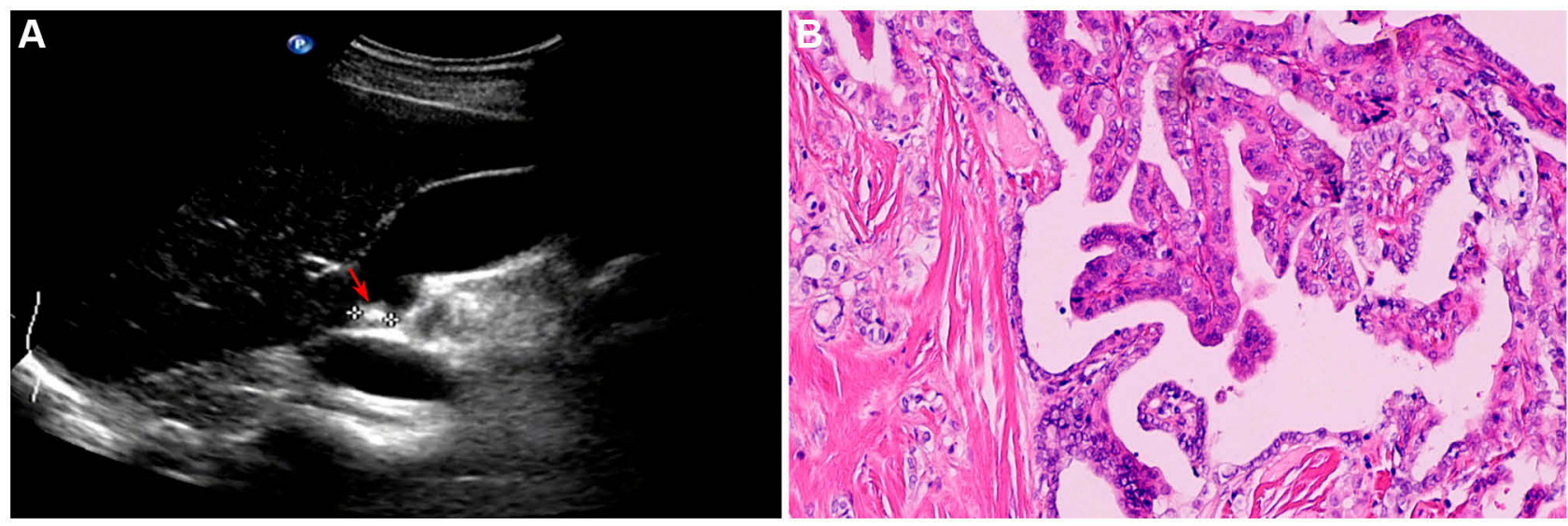

Figure 5 Case 5 pathological figures. (A) A hypoechoic mass can be seen in the left lobe of the thyroid gland, and a punctate lesion with strong echo was seen (arrow). (B) Thyroid biopsy result revealed papillary carcinoma (Hematoxylin \& Eosin staining, $\times 20)$. 
$\mu \mathrm{mol} / \mathrm{L}$, serum calcium of $2.77 \mathrm{mmol} / \mathrm{L}$, serum LDH of 299 $\mathrm{U} / \mathrm{L}$, and serum $\beta 2-\mathrm{MG}$ of $3.9 \mathrm{mg} / \mathrm{L}$. The serum $\mathrm{IgG}$ and serum kappa light chain was $17.37 \mathrm{~g} / \mathrm{L}$ and $12.02 \mathrm{~g} / \mathrm{L}$, respectively. The serum EP and IEP also showed abnormally bowed arcs against IgG and anti-kappa light chain. Bone marrow (BM) aspirate demonstrated up to $13.2 \%$ dysplastic plasma cells, which were proved to be monoclonal plasma cells by flow cytometry. The pathology of the right femur puncture proved the malignant tumor; however, immunological labeling was not ideal and it was necessary to identify the plasma cell tumor and diffuse large B-cell lymphoma. Immunohistochemistry showed CK (-), Vim (-), CD5/6 (-), CK8/18 (-), P63 (-), CK18 (-), CD138 (-), Ki-67 approximately 20\%, CD3 (-), CD5 (-), CD79a (+), CD20 $(+)$, kappa (-), and lambda (-). In view of the current clinical diagnosis of MM, the VCD scheme (bortezomib $1.3 \mathrm{mg} / \mathrm{m}^{2}$ on days $1,4,8$ and 11 , cyclophosphamide $200 \mathrm{mg} / \mathrm{m}^{2}$ on days 1, 2, 3 and 4, and dexamethasone $20 \mathrm{mg}$ on days 1, 2,
4, 5, 8, 9, 11 and 12;) was administered. After four cycles of chemotherapy, the levels of $\mathrm{M}$ protein, IgG heavy chain, and kappa light chain decreased significantly compared to before chemotherapy. However, the patient continued to suffer from pain in the right hip and MRI showed that the soft tissue mass near the right femur was larger than before. A biopsy was performed again and the immunohistochemical markers were supplemented. Finally, the diagnosis of diffuse large B-cell lymphoma was confirmed (Figure 6). Thus, the ultimate diagnoses were MM with type of IgGkappa (D-S stage IIIA and ISS stage II) and diffuse large B-cell lymphoma with the invasion of the paraplastic soft tissue (Ann-Arbor stage II and International Prognostic Index score 3). After two cycles of the R-EPOCH scheme (rituximab $600 \mathrm{mg}$ on day 1 , etoposide $50 \mathrm{mg} / \mathrm{m}^{2}$ on days $1-4$, vindesine $0.4 \mathrm{mg} / \mathrm{m}^{2}$ on days $1-4$, doxorubicin $9 \mathrm{mg}$ / $\mathrm{m} 2$ on days 1-4; cyclophosphamide $750 \mathrm{mg} / \mathrm{m}^{2}$ on day 5 , and prednisone $60 \mathrm{mg} / \mathrm{m}^{2}$ on days 1-5) combined with
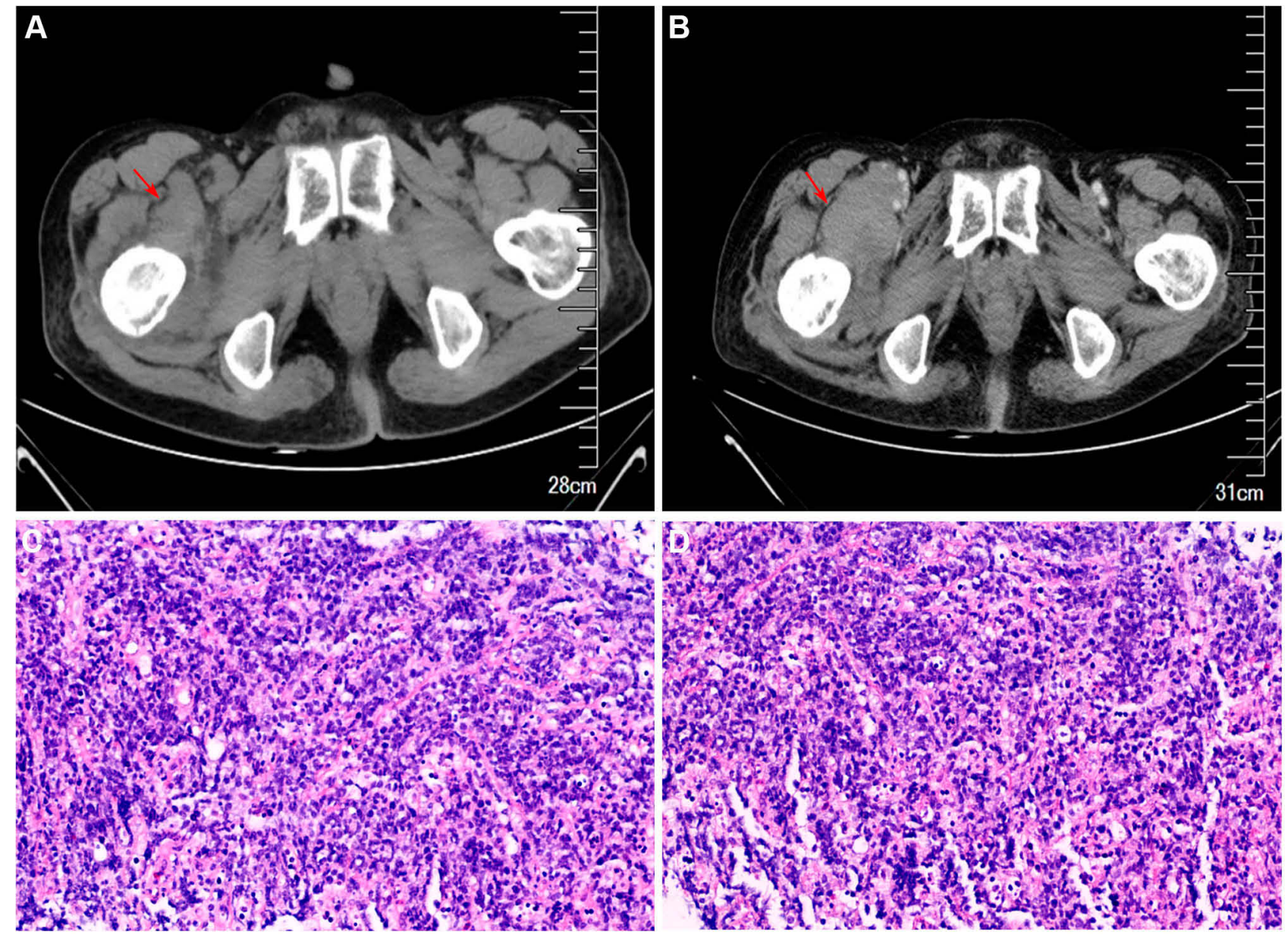

Figure 6 Case 6 pathological figures. (A) The soft tissue mass near the right femur (arrow) at initial diagnosis. (B) After 4 courses of chemotherapy for multiple myeloma, the soft tissue mass near the right femur was significantly larger than that at initial diagnosis. (C) Pathologica biopsy result revealed lymphoma or plasmacytoma at initial diagnosis (Hematoxylin \& Eosin staining, $\times 20$ ). (D) The second pathological biopsy result revealed lymphoma (Hematoxylin \& Eosin staining, $\times 20$ ). 
lenalidomide (25 $\mathrm{mg}$ on days 1-21), a reexamination showed that the size of the soft tissue mass had decreased significantly. The patient is still alive at present with a total survival time of more than 11 months.

\section{Discussion}

The outcomes of this study presented that five cases of immunoglobulin $\mathrm{G}$ ( $\operatorname{IgG}$ ) and one case of kappa light chain; D-S stage: six case of stage III; ISS stage: one case of stage I, two cases of stage II, and three cases of stage III; one case each of gastric cancer (pT2N0MO, stage I), breast cancer (pT1bN0M0, stage I), lung cancer (pT1N0M0, stage I), cervical cancer (stage IB2), thyroid cancer (pT1N0M0, stage I), and diffuse large B-cell lymphoma (Ann-Arbor stage II); three of five patients underwent surgery alone, one patient underwent surgery first and then received chemotherapy at the time of pleural metastasis and the other patient only received radiotherapy; two patients were still alive, three died of progression of MM, and one died of lung cancer. The median survival time was 33.5 months (95\% CI, 14.17 to 59.5months).

Recently, the synchronous presence of multiple myeloma (MM) and other primary malignant tumors (PMTs) are rarely observed in clinical practice. It been reported that MM patients may have an immunologic tolerance for developing solid tumors since these patients have an impaired immune system. Bedides, carcinoembryonic antigen-related cell adhesion molecule 6 (CEACAM6) plays a significant role in $\mathrm{MM}$ and non-hematologic malignancies mentioned above. Some scholars have found that the monoclonal Ig synthesized by two cell types had the same type of light chain and the idiotypic determinant of Ig expression in serum and plasma cells could be detected on the surface of lymphoma cells. Additionally, the possibility of transforming lymphoma cells into plasma cells appears doubtful. In terms of treatment, it depends on the location of the tumor. For patients with MM alongside solid tumors, surgical resection is the first choice, but patients with a late-stage disease or a poor general condition should be treated by a combination of therapeutic approaches.

Considering a 3-fold increase in the prevalence of $\mathrm{MM}^{9,10}$ and with the increased availability of methods with greater sensitivity for screening organs susceptible to MPMTs, there will be an increase of frequency in the identification of MM with sMPMTs in the coming years. However, the precise etiology and pathogenesis of MM with sMPMTs remains poorly understood so for. ${ }^{11}$ In this report, we found only one patient (case 1) who might carry a genetic predisposition because both his father and aunt had died from gastric cancer, while the other five patients did not seem to be involved with factors leading to MPMTs mentioned above.

Generally, it remains unclear whether a MM itself is a risk factor for the incidence of solid neoplasms. ${ }^{12}$ It has also been reported that MM patients may have an immunologic tolerance for developing solid tumors since these patients have an impaired immune system. ${ }^{13}$ Six large MM group studies have shown that MM might be associated with an increased risk of secondary malignancies, including hematologic malignancies and solid cancers. ${ }^{14}$ Therefore, it is suggested that MM may be associated with an increased risk of other malignancies.

There is a similar genetic abnormality between MM and solid tumors. ${ }^{15}$ Carcinoembryonic antigen-related cell adhesion molecule 6 (CEACAM6) plays a significant role in $\mathrm{MM}$ and non-hematologic malignancies mentioned above. The abnormal expression of CEACAM6 is also closely related to tumor progression, recurrence, and prognosis. ${ }^{16,17}$ Mutation of the MYC gene is very high in $\mathrm{MM}^{18}$ and its high expression is also related to the invasiveness of breast cancer. In an experimental study performed on mice, the mutation of threonine 58 , which encodes the MYC protein, to alanine was linked to the development of breast cancer and the mutation of serine 62 to alanine was found to reduce the incidence of breast cancer. ${ }^{19}$ Besides, in this study, there were four patients presented high risk cytogenetic abnormalities among these six participants. However, the specific mechanism remians unknown and should be further research.

The literature on MM alongside other hematologic malignancies is relatively rare. ${ }^{20}$ In case 6 , the two blood diseases both originated from $B$ cells, which could reflect the multidirectional transformation of tumor B cells. Some scholars have found that the monoclonal Ig synthesized by two cell types had the same type of light chain and the idiotypic determinant of Ig expression in serum and plasma cells could be detected on the surface of lymphoma cells. Additionally, the possibility of transforming lymphoma cells into plasma cells appears doubtful. Le Baccon et al Reported that 1q12 heterochromatin is involved in the pathogenesis of NHL and $\mathrm{MM}^{21}$ Their findings indicated that the high frequency of 1q12 rearrangement in NHL and MM is consistent with its pathogenic role, demonstrating the relationship between MM and NHL at the gene level. 
Although similar cases have not been described in this paper, studies have shown that renal cell carcinoma (RCC) is closely related to MM. Previous studies have reported that adipose tissue is the main source of inflammatory mediators, especially IL- $6{ }^{22}$ Moreover, they pointed out the importance of IL-6 in this coexistence, further proving that the two diseases were interrelated.

The choice of treatment depends on the location of the tumor. Recently, for patients with MM alongside solid tumors, surgical resection is the first choice, but patients with a late-stage disease or a poor general condition should be treated by a combination of therapeutic approaches. In this report, for case 3, the patient received MM chemotherapy first after undergoing surgery, then began to alternately receive different chemotherapy regimens for the treatment of multiple myeloma and lung cancer at the time of the pleural metastasis of the lung cancer. After accepting the symptomatic treatments such as blood transfusions and cervical local radical radiotherapy, the patient in case 4 received chemotherapy for MM. While for case 6, after confirming that the patient had multiple myeloma with the presence of synchronous lymphoma, a chemotherapy regimen combining the two diseases was given. However, in terms of the case 6 , the evidence supporting the primary diagnosis of multiple myeloma about the case 6 may be slightly insufficient. Because, it was really difficult to discriminate between primary DLBCL of bone and multiple myeloma/MGUS. Therefore, the diagnosis of multiple myeloma with synchronous lymphoma should be further research in the future.

\section{Highlights}

The synchronous presence of MM and other PMTs are rarely observed in clinical practice. This study aims to analyze several cases of MM and other PMTs in order to improve clinicians' awareness of multiple myeloma (MM) with sMPMTs. This study may be the largest case series on the synchronous presence of multiple myeloma and other PMTs. The results showed that the relationship between synchronous MM and other PMTs remains inconclusive. Therefore, clinicians should improve their understanding of MM with sMPMTs by carrying out multidisciplinary collaboration and a patient-oriented approach to optimize treatment and prolong the survival rates of patients.

\section{Limitations}

Firstly, this trial was only a respective study and not a randomized controlled trial. Secondly, this study was only a single-center trial and the sample size was limited. Thirdly, the study did not design the control group, which should be further researched. Fourthly, there was no any tables about the published case reports of the literature which should be further research in the future. Fifthly, the molecular connection between synchronous MM and other PMTs remains unknown and should be further research.

\section{Conclusion}

The relationship between synchronous $\mathrm{MM}$ and other PMTs remains inconclusive. Clinicians should improve their understanding of MM with sMPMTs by carrying out multidisciplinary collaboration and a patient-oriented approach to optimize treatment and prolong the survival rates of patients.

\section{Ethics Approval}

This study was conducted with approval from the Ethics Committee of our hospital. This study was conducted in accordance with the declaration of Helsinki. All patients have provided written informed consent for the case details to be published.

\section{Acknowledgments}

The authors would like to thank all the patients for their cooperation and the staff at the Henan Institute of Haematology. This study was supported by grants from the National Natural Science Foundation of China (No. 81470287) and the Henan Outstanding Person Foundation (No. 2018JR007).

\section{Author Contributions}

All authors contributed to data analysis, drafting or revising the article, gave final approval of the version to be published, and agree to be accountable for all aspects of the work.

\section{Disclosure}

The authors declare that they have no competing interests in this work.

\section{References}

1. Yoshida N, Tamaoki Y, Baba Y, et al. Incidence and risk factors of synchronous colorectal cancer in patients with esophageal cancer: an analysis of 480 consecutive colonoscopies before surgery. Int $J$ Clin Oncol. 2016;21:1079-1084. doi:10.1007/s10147-016-1015-8

2. Zuo W, Zhu X, Yang J, et al. Bortezomib combined with lenalidomide as the first-line treatment for the rare synchronous occurrence of multiple myeloma and pulmonary adenocarcinoma: a case report. Medicine (Baltimore). 2017;96(1):e5787. doi:10.1097/MD.000000000 0005787 
3. Vennepureddy A, Motilal Nehru V, Liu Y, Mohammad F, Atallah JP. Synchronous diagnosis of multiple myeloma, breast cancer, and monoclonal B-cell lymphocytosis on initial presentation. Case Rep Oncol Med. 2016;2016:7953745.

4. Inoue T, Hasegawa J, Mikuriya K, et al. A case of conversion surgery following combined chemotherapy with molecular targeting agent for unresectable colorectal liver metastases. Gan to Kagaku Ryoho. 2019;46(3):499-501.

5. Mateos MV, San Miguel JF. Management of multiple myeloma in the newly diagnosed patient. Hematol Am Soc Hematol Educ Program. 2017;2017(1):498-507. doi:10.1182/asheducation-2017.1.498

6. Diamond E, Lahoud OB, Landau H. Managing multiple myeloma in elderly patients. Leuk Lymphoma. 2018;59(6):1300-1311. doi:10.10 80/10428194.2017.1365859

7. Richter J, Jagannath S. Society of hematologic oncology state of the art update and next questions: multiple myeloma. Clin Lymphoma Myeloma Leuk. 2018;18(11):693-702. doi:10.1016/j.clml.2018.09.003

8. Blimark CH, Turesson I, Genell A, et al.; Swedish Myeloma Registry. Outcome and survival of myeloma patients diagnosed 2008-2015. Real-world data on 4904 patients from the Swedish Myeloma Registry. Haematologica. 2018;103(3):506-513. doi:10.33 24/haematol.2017.178103

9. Liu Z, Liu C, Guo W, Li S, Bai O. Clinical analysis of 152 cases of multiple primary malignant tumors in 15,398 patients with malignant tumors. PLoS One. 2015;10(5):e0125754. doi:10.1371/journal.pone. 0125754

10. Kehrer M, Koob S, Strauss A, Wirtz DC, Schmolders J. [Multiple myeloma - Current status in diagnostic testing and therapy]. Z Orthop Unfall. 2017;155(5):575-586. Geman. doi:10.1055/s-0043-110224

11. Li QL, Ma JA, Li HP, et al. Synchronous colorectal cancer and multiple myeloma with chest wall involvement: is this a coincidence? Curr Probl Cancer. 2017;41(6):413-418.

12. Kawano Y, Roccaro AM, Ghobrial IM, Azzi J. Multiple myeloma and the immune microenvironment. Curr Cancer Drug Targets. 2017;17(9):806-818. doi:10.2174/1568009617666170214102301

13. Feng X, Zhang L, Acharya C, et al. Targeting CD38 suppresses induction and function of $\mathrm{T}$ regulatory cells to mitigate immunosuppression in multiple myeloma. Clin Cancer Res. 2017;23(15): 4290-4300. doi:10.1158/1078-0432.CCR-16-3192
14. Johnson B, Mahadevan D. Emerging role and targeting of carcinoembryonic antigen-related cell adhesion molecule 6 (CEACAM6) in human malignancies. Clin Cancer Drugs. 2015;2(2):100-111. doi:10. 2174/2212697X02666150602215823

15. Manier S, Salem KZ, Park J, Landau DA, Getz G, Ghobrial IM. Genomic complexity of multiple myeloma and its clinical implications. Nat Rev Clin Oncol. 2017;14(2):100-113. doi:10.10 38/nrclinonc. 2016.122

16. Shi J, Ni Y, Li J, Qiu H, Miao K. Concurrent chronic neutrophilic leukemia blast crisis and multiple myeloma: a case report and literature review. Oncol Lett. 2015;9(5):2208-2210. doi:10.3892/ol.2015. 3043

17. Son SM, Yun J, Lee SH, et al. Therapeutic effect of pHLIP- mediated CEACAM6 Gene silencing in lung adenocarcinoma. Sci Rep. 2019;9 (1):11607. doi:10.1038/s41598-019-48104-5

18. Chandran R, Simon M, Spurgeon SE. Concurrent presentation of hodgkin lymphoma and multiple myeloma. Case Rep Hematol. 2013;2013:398769.

19. Murukutla S, Arora S, Bhatt VR, Kedia S, Popalzai M, Dhar M. Concurrent acute monoblastic leukemia and multiple myeloma in a 66-year-old chemotherapy-naive woman. World J Oncol. 2014;5 (2):68-71. doi:10.14740/wjon722w

20. Deckert J, Wetzel MC, Bartle LM, et al. SAR650984, a novel humanized CD38-targeting antibody, demonstrates potent antitumor activity in models of multiple myeloma and other CD38+ hematologic malignancies. Clin Cancer Res. 2014;20(17):4574-4583. doi:10.11 58/1078-0432.CCR-14-0695

21. Le Baccon P, Leroux D, Dascalescu C, et al. Novel evidence of a role for chromosome 1 pericentric heterochromatin in the pathogenesis of B-cell lymphoma and multiple myeloma. Genes Chromosomes Cancer. 2001;32(3):250-264. doi: 10.1002/gcc.1189

22. Knudsen JG, Gudiksen A, Bertholdt L, et al. Skeletal muscle IL-6 regulates muscle substrate utilization and adipose tissue metabolism during recovery from an acute bout of exercise. PLoS One. 2017;12 (12):e0189301. doi:10.1371/journal.pone.0189301

\section{Publish your work in this journal}

Cancer Management and Research is an international, peer-reviewed open access journal focusing on cancer research and the optimal use of preventative and integrated treatment interventions to achieve improved outcomes, enhanced survival and quality of life for the cancer patient.
The manuscript management system is completely online and includes a very quick and fair peer-review system, which is all easy to use. Visit http://www.dovepress.com/testimonials.php to read real quotes from published authors. 